\title{
Influence of defect shape in quantitative thermography
}

\author{
by A. Stoynova* and B. Bonev**
}

\author{
*Technical University of Sofia, 8, Kliment Ohridski Blvd., Sofia, Bulgaria, ava@ecad.tu-sofia.bg \\ **Technical University of Sofia, 8, Kliment Ohridski Blvd., Sofia, Bulgaria, bonev@ecad.tu-sofia.bg
}

\begin{abstract}
This article examines the impact of the form of hidden defects on their detection and evaluation using flash and lock-in thermography. The influence of the defect shape on information characteristics of quantitative thermography has been studied through digital thermal modeling and simulation. The following information characteristics are analyzed and compared - amplitude and phase contrast, temperature difference between defect and non-defect area, the moment of reaching maximum thermal contrast for different modes of optically active thermography. The results from the model study were experimentally verified using specially designed test specimens with artificial defects. The results obtained were evaluated, compared and analyzed.
\end{abstract}

\section{Introduction}

Lock-in and flash thermography have become increasingly popular in recent years as methods not only for detecting hidden defects, but also for quantifying them. Most studies have focused on determining some of the geometric dimensions of defects - most often the depth at which it is located [1,2]. Determining each of the geometric dimensions of the defects is difficult because it is influenced by many other parameters. For example, the area of the defect directly influences the information parameters, which is used for depth determination (e.g. phase contrast in lock-in thermography) [3]. In recent years, the possibility for 3D sizing of defects has been investigated [4 - 6].

The shape of the defect is one of the influencing factors on the information parameters, used by defect characterization methods. Unlike the geometric dimensions of the defect (width, length etc.), the shape of the defect is not easily characterized by a certain value. This article examines defects of different complex shapes with the same other parameters to investigate the effect of shape.

\section{Experimental}

The study was performed both by simulation of three-dimensional models and by real thermographic measurement of specially designed experimental specimen. Modeling and simulation study was performed by the widely used specialized software for three-dimensional modeling in the field of active thermography ThermoCalc 3D [7].

The processing of the results from both simulation and real measurements was performed using an own developed software product in the MatLab environment, which implements the most used processing methods in active thermography.

For the simulation studies, three-dimensional models of an experimental specimen containing artificial defects with different shapes were created in the ThermoCalc 3D environment. FR4 was selected for the defect-free area material and copper for the defect area material. The defect shapes studied are shown in Fig. 1.

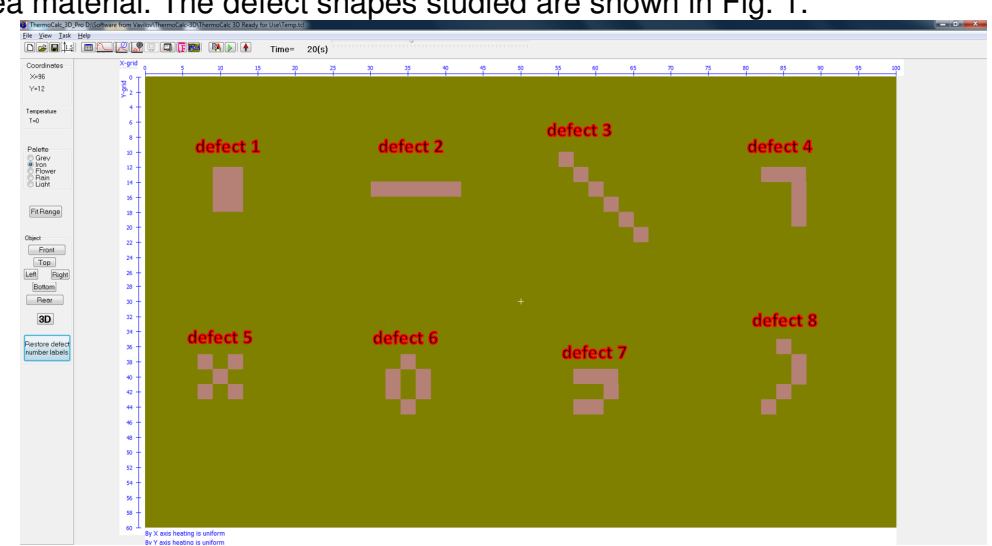

Fig. 1. Created model in the ThermoCalc 3D environment

The simulation study was performed at two modes of active thermography - flash and lock-in thermography at different parameters of thermal stimulation - energy and pulse length (flash thermography), lock-in frequency (lock-in thermography). Studies were carried out for two different values of the thickness and depth of the defect. 
Experimental specimens based on the created models have been prepared and experimental measurements have been performed to verify the results of the simulation study.

The following information parameters used to quantify defects are selected as an indicator to evaluate the impact of the defect shape: temperature difference between defect and non-defect area (flash thermography) $-\Delta T$; time of reaching maximum $\Delta T$ (flash thermography) - $t_{\text {max }}$; amplitude contrast $-\Delta A$; phase contrast (lock-in thermography) - $\Delta \varphi$.

\section{Results}

Fig. 2 and Fig. 3 show the results from the simulation study at flash thermography and lock-in thermography. The results show the significant influence of the defect shape on the information parameters used in defect characterization, which is a prerequisite for a significant error if the specific defect shape is not taken into account.

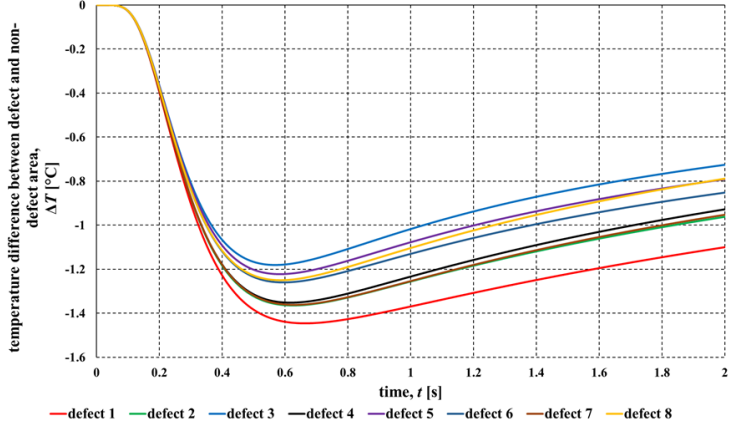

(a) impact of defect shape on $\Delta T$

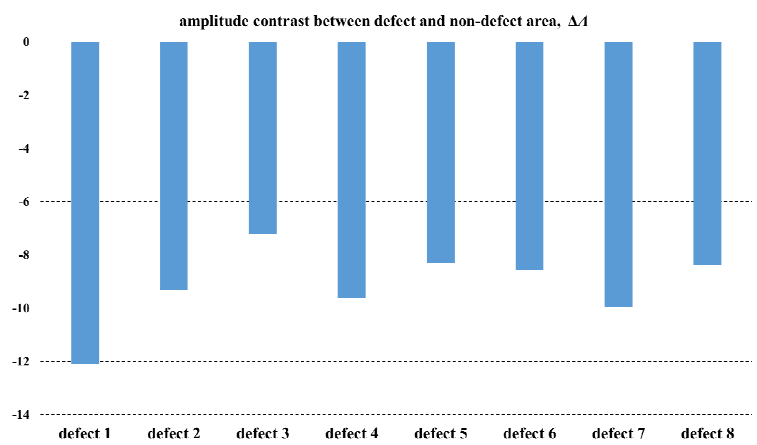

(c) impact of defect shape on $\Delta A$

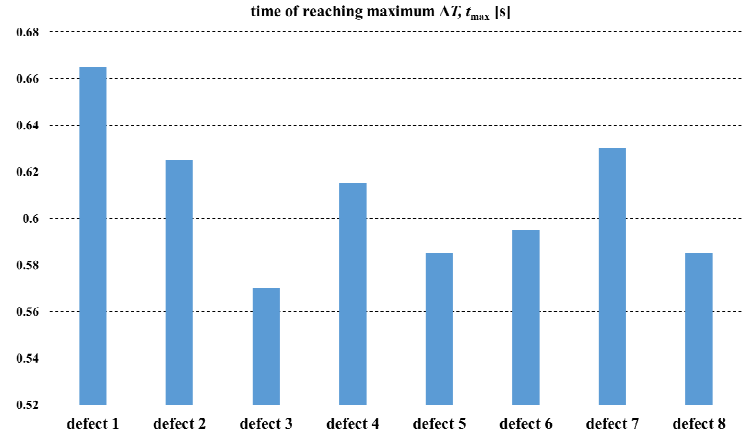

(b) impact of defect shape on $t_{\max }$

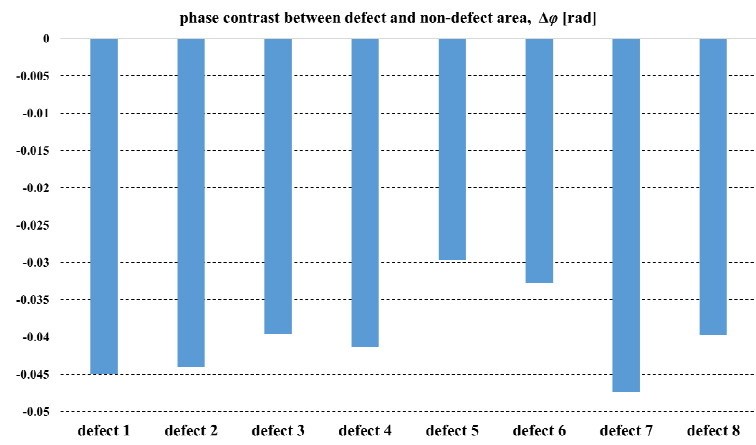

(d) impact of defect shape on $\Delta \varphi$

Fig. 2. Results from simulation study of the models - flash thermography $(a, b)$ and lock-in thermography $(c, d)$

Acknowledgment: The author would like to thank for the support of National Science Fund under which Project No. DN 17/16 the present work was conducted. The authors would like to thank to prof. V. Vavilov, Tomsk polytechnic university, Russia for the support and assistance in thermal modeling and simulation using ThermoCalc 3D software.

\section{REFERENCES}

[1] Ranjit, S., Kang, K., Kim, W., Investigation of Lock-in Infrared Thermography for Evaluation of Subsurface Defects Size and Depth, International Journal of Precision Engineering and Manufacturing. - Vol. 16, pp. 22552264, 2015.

[2] Sharath D., Menaka, M., Balasubramaniam, V., Baldev, R., Defect depth quantification using lock-in thermography, Quantitative InfraRed Thermography Journal. - Vol. 12, pp. 1-16, 2015.

[3] Wallbrink C., Wade S., Jones R., The effect of size on the quantitative estimation of defect depth in steel structures using lock-in thermography, Journal of applied physics. - Vol. 101, p. 104907, 2007.

[4] A. Sirikham, Y. Zhao, H. Liu, Y. Xu, S. Williams and J. Mehnen, Three-dimensional subsurface defect shape reconstruction and visualisation by pulsed thermography, Infrared Physics \& Technology. - Vol. 104, p. 103151, 2020.

[5] S. Pawar and V. Vavilov, Applying the heat conduction-based 3D normalization and thermal tomography to pulsed infrared thermography for defect characterization in composite materials, International Journal of Heat and Mass Transfer. - Vol. 94, pp. 56-65, 2016.

[6] S. Ekanayake, C. Isenberg and R. H. Schmitt, Method for Quantitative 3D Evaluation of Defects in CFRP Using Active Lock-in Thermography, Procedia CIRP. - Vol. 66, pp. 254-258, 2017.

[7] V. Vavilov, "Three-dimensional analysis of transient thermal NDT problems by data simulation and processing," Proceedings of SPIE - The International Society for Optical Engineering, DOI: 10.1117/12.381546, 2000. 